Revue d'histoire de l'Amérique française

REVUE D.HISTOIRE DE L'AMÉRIQUE FRANÇAISE

\title{
Les pièges de la judiciarisation de l'histoire autochtone
}

\section{Alain Beaulieu}

Volume 53, numéro 4, printemps 2000

Histoire des Premières Nations : nouvelles lectures et nouveaux problèmes

URI : https://id.erudit.org/iderudit/005323ar

DOI : https://doi.org/10.7202/005323ar

Aller au sommaire du numéro

Éditeur(s)

Institut d'histoire de l'Amérique française

ISSN

0035-2357 (imprimé)

1492-1383 (numérique)

Découvrir la revue

\section{Citer cet article}

Beaulieu, A. (2000). Les pièges de la judiciarisation de l'histoire autochtone.

Revue d'histoire de l'Amérique française, 53(4), 541-551.

https://doi.org/10.7202/005323ar d'utilisation que vous pouvez consulter en ligne.

https://apropos.erudit.org/fr/usagers/politique-dutilisation/ 


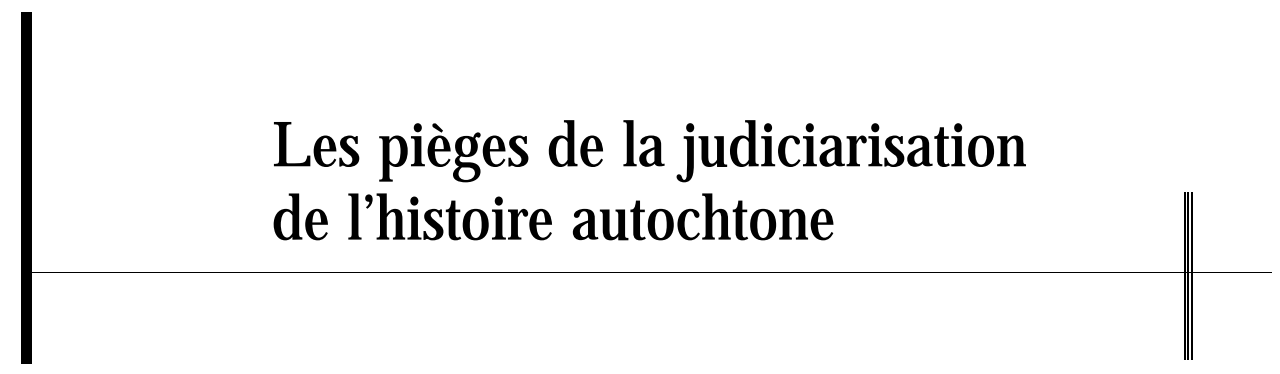

ALAIN BEAULIEU

Département d'histoire

Université du Québec à M ontréal

\begin{abstract}
C omment le contexte juridique influence-t-il I'historiographie récente se rapportant aux Autochtones? C'est sous cet angle que j'ai décidé d'inscrire ma participation à cette «table ronde» organisée par la Revue d'histoire de l'Amérique française Depuis quelques années, les tribunaux jouent un rôle crucial dans le processus de revendication autochtone au Canada. De plus en plus les Amérindiens et les Inuits, mais aussi les gouvernements - fédéral ou provinciaux - leur demandent de déterminer la portée exacte des droits autochtones qu'il s'agisse de droits ancestraux, de droits issus de traités ou de droits découlant de la Proclamation royale de 1763. Dans la plupart des cas, l'histoire est appelée à la «barre des témoins», soit pour fonder historiquement les droits en question ou, au contraire, pour les nier ou en limiter la portée.

Ce recours de plus en plus fréquent à l'histoire dans l'arène juridique soulève plusieurs problèmes. Des problèmes éthiques et moraux, bien sûr : les historiens doivent-ils mettre leurs connaissances et leur expertise au service d'une cause? devraient-ils accepter d'effectuer des recherches historiques en sachant qu'elles seront utilisées pour contester des droits revendiqués par les Autochtones? dans quelle mesure ceux qui participent à ces débats sont-ils des «historiens de service», qui acceptent de ne chercher que les éléments historiques qui appuient une thèse qu'on leur soumet? Des problèmes épistémologiques aussi : comment les balises
\end{abstract}


imposées par le cadre juridique orientent-elles les recherches historiques, même celles qui ne sont pas préparées dans un cadre juridique? comment le contexte juridique favorise-t-il l'émergence de nouveaux «tabous», écartant certaines questions jugées trop délicates en raison des incidences qu'elles pourraient avoir sur les revendications autochtones? J'aborderai ici ces questions à travers deux exemples: 1) celui du territoire et de l'identité des nations qui l'habitent et 2) celui des traités conclus avec certains Amérindiens au moment de la Conquête. Ces deux exemples, très polémiques, me semblent particulièrement révélateurs des pièges posés par la judiciarisation des recherches historiques sur les Autochtones. Je ne prétends cependant pas aborder ces questions de manière pleinement impartiale et totalement au-dessus de la mêlée - à supposer que cela soit possible - , car au cours des dernières années, j'ai participé à ce genre de recherches historiques dans un contexte juridique, tantôt pour le gouvernement du Québec, tantôt pour le gouvernement fédéral et tantôt pour des Amérindiens Certains diront que j'ai les qualités d'un parfait mercenaire - on me l'a déjà laissé entendre. Pour ma part, je vois ces collaborations avec des camps opposés comme la condition essentielle d'une véritable indépendance intellectuelle. Elles m'ont aussi permis de prendre conscience d'un fait que l'on a trop souvent tendance à occulter: la judiciarisation de I'histoire présente les mêmes pièges dans un camp comme dans l'autre.

une identité figée ou mouvante?

Dans la logique juridique anglo-saxonne, les droits ancestraux des Amérindiens sur le territoire découlent d'une occupation immémoriale et continue des lieux. Pour faire valoir et reconnaître ses droits ancestraux sur un territoire donné, une nation autochtone doit donc pouwir établir sa filiation avec les Amérindiens rencontrés à cet endroit par les premiers Européens Cette logique juridique n'est pas nouvelle, puisqu'on la voit à l'œuvre dès les premières années qui suivent la conquête de la Nouvelle-France, mais elle a été précisée par la jurisprudence des dernières années'. Elle est au cœur des préoccupations de ceux qui entreprennent des recherches historiques sur l'occupation du territoire et imprime sa marque sur la manière de penser cette question.

Une des conséquences les plus manifestes de la logique juridique des droits ancestraux est de favoriser la reconstitution d'une image extrême-

1. Voir, à ce sujet, l'étude de Renée Dupuis, Tribus, peuples et nations: les nouveaux enjeux des revendications autochtones au Canada (Montréal, Boréal, 1997). 
ment statique de l'occupation du teritoire et de l'identité des nations amérindiennes qui l'ont habité au cours de l'histoire. Pour répondre pleinement aux critères juridiques et éviter que l'histoire ne devienne un outil pour nier l'existence de droits, il faut gommer autant que possible les phénomènes de migration et d'amalgame de populations qui auraient pu survenir à la suite de guerres ou d'épidémies

Cette orientation donnée par le cadre juridique s'est manifestée ouvertement en 1996, à la suite d'un reportage de Radio-Canada, qui faisait état de recherches entreprises depuis une quinzaine d'années par le gouvernement québécois et $\mathrm{H}$ ydro-Québec. Selon le journaliste Paul Toutant, ces recherches concluaient que les Atikamekw et les Montagnais, à qui le gouvernement avait fait une offre de règlement en 1994, étaient en fait des arrivants de «fraîche date» en territoire québécois et ne pouvaient donc pas revendiquer des droits ancestraux. Les Atikamekw et les Montagnais étaient bien «es descendants d'Amérindiens», expliqua le journaliste, «mais installés sur le territoire apRès l'arrivée des Européen $s^{2} \gg$. Les historiens Maurice Ratelle, à l'emploi du ministère des Ressources naturelles, et Russel Bouchard, chercheur indépendant, étaient venus confirmer brièvement à la caméra les affirmations du journaliste de Radio-Canada.

Dans le cas des Atikamekw, le scoop de Radio-Canada n'apportait rien de vraiment nouveau. L'hypothèse concernant leur disparition et leur remplacement par des Amérindiens venus de la région des Grands Lacs était connue depuis longtemps, car l'anthropologue N orman Clermont I'avait énoncée dès $1977^{3}$. Par contre, l'élargissement de cette hypothèse aux Montagnais était relativement nouvelle et encore peu connue. Le reportage de Radio-Canada, qui touchait une corde sensible, eut des échos dans le milieu de la recherche autochtone. II provoqua surtout des

2. Le texte de ce reportage est reproduit dans Recherches amérindiennes au Québec, 26,2 (automne 1996): 83.

3. Norman Clermont, Ma femme, ma hache et mon couteau croche: deux siècles d'histoire à Weymontachie (Québec, Ministère des Affaires culturelles, 1977). Clermont avait été précédé, deux siècles plus tôt, par I'historien jésuite Charlevoix qui affirmait: «ll y en avait [des Amérindiens], qui descendaient dans la colonie des quartiers du nord, quelquefois par le Saguenay, et plus souvent par les Trois-Rivières, et dont on n'entend plus parler depuis longtemps. Tels étaient entr'autres les Attikamegues: ces Sauvages venaient de fort loin, et ils étaient environnés de plusieurs autres peuples [... ]. Presque tous ont été détruits par le fer des Iroquois, ou par les maladies, suite de la misère, où la crainte de ces barbares les avaient réduits. » Charlevoix, Histoire et description generale de la Nouvelle France, Avec le Journal historique d'un Voyage fait par ordre du Roi dans I'Amerique Septentrionale (Paris, Nyon Fils, 1744), III : 186. 
réactions outrées chez certains chercheurs, qui critiquèrent la manière dont le gouvemement québécois et la société d'État tentaient de manipuler l'histoire pour appuyer leur position juridique niant l'existence de droits ancestraux pour les Autochtones vivant au Québec.

Je ne retiendrai ici, à titre d'exemple, que les propos de l'anthropologue Paul Charest, notamment ses remarques à l'égard des travaux préparés par l'historien Raynald Parent qui, en 1985, avait déposé au gouvernement du Québec un volumineux rapport sur l'histoire des Atikamekw et des Montagnais arant $1760^{4}$. Dans ce rapport, Parent soutenait à la fois la thèse d'une disparition des Atikamekw et celle de la continuité dans l'occupation du teritoire par les M ontagnais Les propos de Charest, d'abord publiés dans le journal Le Solei ${ }^{5}$, critiquaient Parent pour avoir conclu à la disparition des Atikamekw. Comme la plupart des historiens, Parent aurait été insensible aux lacunes des sources historiques lorsqu'il s'agissait de juger de l'occupation du teritoire: «les seules sources historiques utilisées par Parent [...] comportent des limites méthodologiques, que les historiens oublient souvent de rappeler, et elles sont insuffisantes pour que leur hypothèse de la disparition des Atikamekw soit acceptable par la communauté scientifique québécoise et par le gouvernement du Québec. » Curieusement, les limites méthodologiques des mêmes sources étaient tout à fait occultées pour l'autre thèse de Parent, celle concernant la continuité dans l'occupation du territoire par les Montagnais Critiqué pour avoir affirmé la disparition des Atikamekw, le rapport de Parent devenait soudain «une étude très fouillée», dans laquelle on retrouvait «de très nombreuses cartes» appuyant la démonstration d'une «continuité d'occupation par tous les groupes montagnais du Québec de toutes les parties de leur teritoire».

Mon intention n'est pas de déterminer ici qui a tort ou raison dans ce débat. Ce qui m'intéresse essentiellement, c'est le réflexe critique qui se manifeste dès qu'on aborde la question territoriale autochtone. Les mêmes sources n'ont apparemment pas le même poids si elles servent à démontrer la continuité dans l'occupation du territoire ou si elles témoi-

4. Raynald Parent, Histoire des Amérindiens, du Saint-M auricejusqu'au Labrador : de la préhistoire à 1760 (Gouvernement du Québec), 4 vol.

5. Paul Charest, «La supposée disparition des A tikamekw et des M ontagnais», Le Soleil, lundi 25 mars 1996; texte reproduit dans Recherches amérindiennes au Québec, 26,2 (automne 1996): 84 85. Je cite ici le texte de Paul Charest par commodité, car il est un des rares à avoir publié ses critiques à la suite du reportage de Radio-Canada. Loin d'être isolée, sa manière d'aborder la question territoriale me semble, au contraire, très répandue dans le milieu universitaire. 
gnent au contraire d'une transformation importante dans cette occupation. Un même historien peut à la fois, dans un seul rapport, être un chercheur capable de produire une «étude très fouillée», lorsque ses conclusions sont favorables aux revendications de certains Amérindiens et se révéler peu sensible aux lacunes de ses sources, lorsque sa démonstration mine les prétentions d'autres groupes autochtones N'aurait-on pas tendance, dans le milieu de la recherche historique sur les Amérindiens, à juger d'abord et avant tout certains travaux en fonction de leur portée politique, plutôt qu'à partir de leur méthode et de la valeur de leur argumentation?

C'est en tout cas l'impression générale qui se dégage de la manière dont la revue Recherches amérindiennes au Québec, par l'entremise de Pierre Trudel, a présenté le débat soulevé par le reportage de RadioCanada. Le titre donnait déjà le ton: «Des Québécois menacés de disparaitre font disparaitre des Atikamekw et des Montagnaiś... » On ne pouvait être plus clair sur le sort qui attendait ceux qui chercheraient à démontrer qu'une nation autochtone avait été décimée après le contact avec les Européens. Implicitement, les chercheurs venus témoigner dans le reportage de Radio-Canada étaient associés à ceux qui contribuaient au «processus de négation de l'Autre» dans «le cadre de conflits territoriauX», tandis que leurs critiques faisaient figure de "scientifiques», soucieux de rétablir les faits et d'apporter les nuances nécessaires?. Le contexte juridique et la classification qu'il engendre entre des «bons» et des «méchants», entre des défenseurs des opprimés et des spécialistes de la négation de l'Autre rendent très délicate toute réflexion historique sur I'occupation du territoire par les Autochtones. Envisager la disparition de certaines nations fait maintenant partie des nouveaux tabous de la recherche historique sur les Amérindiens Dans le contexte juridique actuel, les peuples n'ont plus le «droit» de disparaitre ou de se fondre dans un nouveau groupe.

Si la plupart des chercheurs furent prompts à critiquer les manipulations de I'histoire auxquelles se seraient prêtés les chercheurs associés au gouvernement du Québec, bien peu par contre s'interrogèrent publiquement sur les effets que pouvait avoir la réalisation de recherches historiques sur l'occupation du territoire dans une optique

6. Recherches amérindiennes au Québec, 26,2 (automne 1996): 82-83.

7. Ibid., 82. Les historiens Ratelle et Bouchard étaient invités à répondre à leurs critiques, mais leur condamnation semblait déjà prononcée. 
essentiellement favorable aux Autochtones. Après tout, pourquoi l'association avec une thèse juridique - la négation des droits ancestraux était-elle suspecte sur le plan scientifique, alors qu'un lien tout aussi évident avec la thèse inverse - celle de la reconnaissance des droits ancestraux - ne provoquait aucune réaction critique? La noblesse de la cause suffirait-elle à garantir la rigueur de la démarche et à immuniser les chercheurs contre tout biais idéologique?

Pour le moment, l'attitude critique des universitaires semble se résumer à objectiver partiellement les conditions de production du discours historique sur ces questions Une véritable attitude critique ne devraitelle pas plutôt objectiver pleinement les contextes de production du discours sur ces questions? La lucidité partielle, dirigée principalement contre les travaux qui favorisent les thèses juridiques gouvernementales, est commode, dans la mesure où elle conforte certains chercheurs dans leur image de défenseurs des opprimés, mais elle est loin d'ouvrir la voie à une meilleure connaissance de la réalité historique.

L'incapacité à envisager sérieusement la possibilité de la disparition d'une nation autochtone me semble tout aussi problématique que la volonté de démontrer absolument des phénomènes de migrations. D'un point de vue strictement méthodologique, ces prémisses opposées risquent fort de donner des résultats tout aussi biaisés Ceux qui ont vraiment côtoyé la documentation historique sur ces questions wous diront sans doute qu'elle est très souvent ambiguë. Et, à mon æis, cette ambiguïté ne joue pas à sens unique: si elle permet rarement d'établir avec certitude la disparition d'une nation autochtone, elle laisse aussi planer de sérieux doutes sur la continuité dans l'occupation du territoire par certains groupes. Malheureusement, bien peu de chercheurs - des deux camps confondus - sont prêts à assumer publiquement cette réalité.

de nouveaux dogmes

En 1982, lors du rapatriement unilatéral de la constitution canadienne, le gouvernement fédéral y inscrivit notamment des protections particulières pour les droits issus de traités. Cela engendra, principalement au Québec et dans les Maritimes un engouement sans précédent pour la recherche de traités anciens, car les engagements pris à ces occasions étaient encore potentiellement porteurs de droits. La décision récente de la Cour suprême du Canada dans l'affaire Marshall, décision qui reconnaissait l'existence de droits de pêche aux Micmacs en vertu de traités 
conclus en 1760, a rappelé, si besoin était, à quel point ces questions sont d'actualité et très émotives.

L'étude des traités anciens dans un contexte juridique soulève des problèmes similaires à ceux relevés pour la question teritoriale. Je prendrai ici pour exemple la polémique entourant la décision rendue par la Cour suprême du Canada dans l'affaire Sioui, en 1990. Dans ce jugement, rédigé par le juge Antonio Lamer, la Cour donnait valeur de traité à un bref document que le général James Murray avait remis aux Hurons de Lorette, le 5 septembre 1760. Depuis, certains historiens ont critiqué l'interprétation du juge Lamer, exprimant leur désaccord sur le sens qu'il fallait donner à ce document. Bien que je considèe que les archives démontrent que des traités furent conclus, au moment de la Conquête, entre les Britanniques et les Amérindiens de la vallée du Saint-Laurent, je fais partie de ceux qui jugent que le document retenu par la Cour n'entre pas dans cette catégorie. Mais il n'est pas nécessaire ici de trancher le débat à savoir s'il s'agit d'un sauf-conduit ou d'un traité pour examiner les effets de la judiciarisation dans l'étude de documents historiques

Une des conséquences les plus évidentes de cette judiciarisation est de figer les interprétations de la Cour suprême - lorsqu'elles sont favorables aux Amérindiens - en nouveaux dogmes historiques, qui deviennent autant de symboles de la reconnaissance des droits autochtones Depuis que le plus haut tribunal du pays a décrété que le document du 5 septembre 1760 avait valeur de traité, il est devenu suspect d'en parler comme d'un simple sauf-conduit. Examiner arec réserve l'interprétation historique de la Cour suprême, c'est afficher ses couleurs, celles d'un anti-Autochtone, pour ne pas dire d'un raciste. À preuve, le compte rendu fait par Rémi Savard du livre de Denis Vaugeois (La fin des alliances franco-indiennes ${ }^{8}$ ) où les fondements historiques de la décision du juge Lamer sont vivement critiqués La conclusion du compte rendu de Rémi Savard est particulièrement révélatrice des attaques et des propos qui circulent «en coulisses». À Denis Vaugeois qui affirmait avoir fait son livre «librement, en refusant de servir une cause, ou tout simplement d'être "politically correct"», Rémi Savard répliquait: «Le racisme c'est parfois comme l'alcoolisme: moins on s'en croit atteint, plus on a de chances de l'être9.»

8 Denis Vaugeois, La fin des alliances franco-indiennes: enquête sur un sauf-conduit de 1760 devenu un traité en 1990 (M ontréal, Boréal, 1995).

9. Rémi Savard, «Commentaire sur La fin des alliances franco-indiennes... », Recherches amérindiennes au Québec, 26,1 (printemps 1996): 80. Rares sont les chercheurs qui se laissent aller à ce 
Qu'on soit ou non d'accord avec les opinions politiques de Denis Vaugeois, qui ne les a jamais dissimulées, ou avec ses critiques à l'égard du jugement de la Cour suprême importe peu ici. Ce que je trouve inquiétant dans les propos de Rémi Sarard, c'est l'intransigeance qu'ils reflètent. L'accusation de racisme est lourde de conséquence: est-ce à dire qu'il ne faut plus remettre en question, dans une perspective historique, des décisions des tribunaux qui sont favorables aux thèses défendues par les Amérindiens? est-ce à dire que l'on ne peut plus faire I'histoire de cette période sans dorénarant répéter à l'unisson qu'un traité fut conclu le 5 septembre 1760 entre les Hurons de Lorette et les Britanniques? est-ce la seule façon d'éviter le banc des accusés dans un procès d'intention?

L'intransigeance n'est évidemment pas exclusive à un camp et elle peut revêtir d'autres formes que l'insulte. La reconnaissance de droits spécifiques aux Autochtones - souvent perçus comme des privilèges auxquels n'a pas accès le reste de la société - constitue un terrain propice aux préjugés. Je retiendrai ici les remarques récentes de l'historien Nelson-Martin Dawson, dont les efforts pour tourner en dérision le «traité Murray » me semblent refléter une attitude fort répandue à l'égard de la reconnaissance de droits particuliers pour certaines nations amérindiennes. Qualifiant le document remis aux Hurons de «simple billet de laissez-passer signé par un gouverneur britannique», il écrivait: «Avoir su que de tels laissez-passer pouvaient avoir une aussi longue portée, j'aurais certainement précieusement conservé le laissez-passer que mon directeur, en secondaire IV, m'avait signé afin que j'aie un accès illimité à la bibliothèque de mon école. Je pourrais le faire valoir aujourd'hui, ruse de Sioux, comme un permis me donnant accès illimité à toutes les bibliothèques et centres d'archives du pays, autorisation accordée par mon ministre de l'Éducation d'alors, par la voie de l'un de ses représentants ${ }^{10}$ !»

Tourner en dérision le document du 5 septembre 1760 ou, au contraire, défendre l'infaillibilité de l'interprétation de la Cour suprême

genre d'accusation en public. Habituellement, on se contente du procès d'intention, comme le faisait Paul Charest, en 1996, dans l'article publié à la suite du reportage de Radio-Canada: «Pourquoi s'acharne-t-on tant à démontrer la disparition de certains groupes ou la non-existence de certains traités? Est-ce là souci des faits historiques ou de démontrer que les Amérindiens ne sont pas différents de nous et que les gouvernements ne devraient pas se préoccuper de leurs droits de premiers occupants?» Paul Charest, «La supposée disparition des Atikamekw et des Montagnais», Recherches amérindiennes au Québec, 26,2 (automne 1996): 85.

10. Le Devoir, 30 mai 1999. 
conduisent au même cul-de-sac historique. Dans un cas comme dans l'autre, les enjeux qui se profilent derrière ce document finissent par occulter les conditions dans lesquelles il fut produit. En associant des droits à certaines pièces d'archives, le contexte juridique leur confère une charge émotive qui complique considérablement leur analyse dans une perspective strictement historique. La fixation sur les enjeux contemporains détourne l'attention de ce qui devrait être, selon moi, l'objectif premier des historiens: comprendre et expliquer l'importance et le sens de ce document de 1760 pour les acteurs de cette époque.

Même si on arrive à la conclusion que le document du 5 septembre 1760 n'est pas un traité et que les circonstances qui ont entouré sa rédaction ne peuvent pas lui donner cette valeur, il n'en reste pas moins qu'il s'agit d'un document important dans l'histoire de la communauté huronne, un document qu'il faut éviter de banaliser ou de ridiculiser, même à titre de sauf-conduit. $\mathrm{Ce}$ «billet» fut remis aux Hurons à un moment particulièrement troublé de leur histoire. La défaite des Français, leurs alliés traditionnels, était imminente et suscitait certainement plusieurs inquiétudes: quelle attitude allaient adopter les nouveaux maîtres du Canada à l'égard des anciens alliés autochtones des Français? Quel sort les attendait une fois la France défaite en Amérique? Les Hurons pouvaient d'autant plus craindre les représailles des Britanniques qu'ils avaient abandonné leur village de la région de Québec, après la bataille des Plaines d'Abraham, pour suivre les troupes françaises, qui s'étaient repliées vers Montréal. Une chose est sûre: la rencontre avec le général britannique marqua les Hurons; les anciens conservaient encore, un demi-siècle plus tard, un souvenir précis de l'événement. On pourrait aussi s'interroger sur le sens et la portée des actions accomplies par Murray, qui prit alors les Hurons sous sa protection. Le geste était-il vraiment banal? J'en doute. Bref, au lieu d'examiner ce document en fonction des droits qu'il peut conférer aujourd'hui, les historiens pourraient faire une contribution plus importante en l'étudiant dans une véritalde perspective historique.

que faire?

Comment éviter, à tout le moins partiellement, les pièges de la judiciarisation de l'histoire autochtone? D'abord, je crois, en s'efforçant d'objectiver globalement - et non pas partiellement - le contexte de production du discours historique sur des thèmes ayant des incidences juridiques. II est beaucoup trop simpliste de prétendre - ou de suggérer - 
que les risques de manipulation de l'histoire à des fins politiques ou juridiques se concentrent dans un seul camp. Le contexte juridique risque de produire les mêmes effets pervers sur la démarche historique, que les chercheurs utilisent I'histoire pour défendre les revendications autochtones ou pour les nier.

L'objectivation globale du discours historique sur des questions litigieuses (le territoire, les traités, la Proclamation royale, la Loi sur les Indiens...) constitue un premier pas vers une étape qui me semble encore plus fondamentale: I'objectivation par chaque historien du contexte de production de ses propres recherches, afin de prendre conscience de l'orientation que ce contexte peut donner à l'analyse historique. Cette objectivation personnelle est sans doute la plus difficile à réaliser, non pas en raison du manque d'intégrité des chercheurs, mais plutôt parce qu'il est plus sécurisant d'intenter un procès d'intention aux autres que de réfléchir sur sa propre pratique et ses fondements idéologiques. Exercer sa lucidité envers les autres n'implique aucune remise en question, la tourner vers soi, oui.

Le piège le plus pervers de la judiciarisation de l'histoire - dans la mesure où ceux qui y tombent n'ont pas vraiment conscience d'être pris au piège ou s'y trouvent confortables - est celui qui consiste à prendre fait et cause pour un camp et à juger. Ce piège, celui de l'histoire militante, enferme les historiens dans une logique juridique précise et les conduit à ne chercher dans I'histoire que les éléments qui viennent appuyer les positions défendues par l'une ou l'autre des parties en présence, à occulter les éléments contradictoires ou encore à chercher coûte que coûte des arguments pour en réduire la portée. Selon moi, la diversité, la complexité, la richesse, mais aussi les lacunes de la documentation historique ne se prêtent pas aux conclusions réductionnistes constamment favorables ou défavorables aux revendications autochtones. Les chercheurs devraient plutôt accepter d'emblée que la documentation sur laquelle ils travaillent puisse parfois les conduire à des conclusions qui vont à l'encontre de leurs sympathies idéologiques ou des attentes de leurs clients.

Enfin, au lieu de chercher absolument à faire entrer I'histoire dans un moule juridique précis - ce qui suppose que les chercheurs l'acceptent, à tout le moins implicitement, comme prémisse de base pour leus travaux -, ne faudrait-il pas plutôt critiquer ouvertement les balises posées par le droit? Bien sûr, les critères juridiques sont à la base de la reconnaissance de droits particuliers pour les Autochtones, mais ils sont 
aussi extrêmement discriminatoires, car ils imposent des conditions qui démarquent les nations autochtones entre elles. Pourquoi, par exemple, une nation autochtone installée dans la vallée du Saint-Laurent à I'arrivée de Champlain aurait-elle plus de droits qu'une autre - comme les Hurons - qui s'y installent quelques années plus tard? Au lieu d'accuser certains historiens de chercher à démontrer la coupure dans l'occupation du territoire, ne pourrait-on pas critiquer les critères juridiques qui colorent moralement certaines interprétations historiques? Après tout, les phénomènes de migration sont courants dans l'histoire de l'humanité. L'histoire du peuplement de l'Amérique n'est-elle pas faite d'une série de migrations? Ne pourrait-on pas aussi se demander pourquoi une nation autochtone derrait avoir plus de droits qu'une autre parce que ses représentants ont eu la chance de rencontrer un général britannique en 1760 et d'obtenir de sa part un document la plaçant à l'abri de représailles? Tous les Amérindiens de la vallée du Saint-Laurent n'ont-ils pas été intégrés au même moment et sensiblement de la même manière à l'empire britannique? Ne devraient-ils pas être traités uniformément par le système judiciaire canadien?

À bien y penser, par les balises qu'il impose aux recherches historiques, le cadre juridique ne serait-il pas un des fondements de la négation de l'Autre? 